ISSN: 2600-5859

\title{
Estratificación del pie diabético en el Hospital General Guasmo Sur período 2018 - 2019
}

\section{Stratification of the diabetic foot in the General Hospital South Guasmo $2018-2019$ period}

\author{
Katiuska Patricia Cervantes Moyano. ${ }^{1}$, Alexandra Elizabeth Delgado Rivera. ${ }^{2}$, \\ Karen Katherine Reyes Murillo. ${ }^{3}$ \& Lida Stefania Veloz Estrada. ${ }^{4}$
}

\section{Recibido:05-01-2020 / Revisado:22-01-2020 /Aceptado: 03-02-2020/ Publicado: 05-03-2020}

\begin{abstract}
DOI: https://doi.org/10.33262/concienciadigital.v3i1.2.1167

Background: The diabetic foot is the clinical entity that is represented as the main complication of diabetes, $20 \%$ of diabetics will need an annual hospitalization and of this $25 \%$ is due to the diabetic foot. The main objective of the study was to present and establish the demographic characteristics by means of classification scales of the diabetic foot in the patients who go to the Emergency Department of the General Hospital South Guasmo. Methods: A retrospective, descriptive, observational study was performed in patients with diabetic foot during the year 2018 and 2019. Age, gender, evolution time to present with diabetic foot, clinical characteristics according to the Wagner and Texas scale were analyzed. Microsoft Excel was used and the information was processed to obtain percentages of qualitative and quantitative variables, Pearson's correlation index was used for each classification. Results: 70 patients with diabetic foot were evaluated, $60 \%$ male, predominant age group of 51 to 60 years with $36 \%$. Type of diabetes mellitus 2 with $86 \%$, the evolution time for clinical presentation of the diabetic foot most frequently from 16 to 20 years with 36\%. The Texas and Wagner scale were used for clinical characteristics, mortality rates were in favor with a higher percentage for the Wagner scale with $17 \%$ vs. $5 \%$ for Texas. Conclusions: Staging of the diabetic foot according to the scales used, and

\footnotetext{
${ }^{1}$ Centro de Salud las Malvinas. Ventanas, Ecuador. Email: kat_cervants@ hotmail.com

${ }^{2}$ Consultorio Médico SIVIMED. Guayaquil, Ecuador. Email: adelgadorczs5@gmail.com

${ }^{3}$ Hospital de Especialidades Teodoro Maldonado Carbo. Guayaquil, Ecuador. Email: karen_reyes_m@hotmail.com

${ }^{4}$ Centro de Salud tipo A Yatuvi. Bolívar, Ecuador. Email: velozstefania010@gmail.com
} 
the clinical characteristics found is of medical importance, health promotion, prevention of diabetes mellitus and good management of complications is decisive from the first level of care in Health.

Keywords: Diabetes, diabetic foot, risk factors, complications.

\section{Resumen}

Antecedentes: El pie diabético es la entidad clínica que se representa como la principal complicación de la diabetes, un 20\% de los diabéticos necesitará una internación anual y de esta el $25 \%$ es a causa del pie diabético. El objetivo principal del estudio fue presentar y establecer las características demográficas mediante escalas de clasificación del pie diabético en los pacientes que acuden al departamento de Emergencias del Hospital General Guasmo Sur. Métodos: Se realizó un estudio retrospectivo, descriptivo, observacional en pacientes con pie diabético durante el año 2018 y 2019. Se analizó edad, género, tiempo de evolución para presentar pie diabético, características clínicas según la escala de Wagner y Texas. Se usó Microsoft Excel y se procesó la información para obtención de porcentajes de variables cualitativa y cuantitativa, se empleó el índice de correlación de Pearson para cada clasificación. Resultados: Se evaluó 70 pacientes con pie diabético, de género masculino el 60\%, grupo etario predominante de 51 a 60 años con el $36 \%$. Tipo de diabetes mellitus 2 con el 86\%, el tiempo de evolución para presentación clínica del pie diabético con mayor frecuencia de 16 a 20 años con el 36\%. Se usó la escala de Texas y Wagner para las características clínicas, las tasas de mortalidad se encontraron a favor con mayor porcentaje para la escala de Wagner con el 17\% vs 5\% para Texas. Conclusiones: La estadificación del pie diabético de acuerdo a las escalas empleadas, y las características clínicas encontradas tiene importancia médica, la promoción de salud, la prevención de la diabetes mellitus y un buen manejo de las complicaciones es decisivo desde el primer nivel de atención en salud.

Palabras claves: Diabetes, pie diabético, factores de riesgo, complicaciones.

\section{Introducción}

La diabetes mellitus es responsable de la pérdida de años de vida y disminución de la productividad, sufrimiento físico y emocional y considerable costos médicos y sociales. Las complicaciones crónicas de esta enfermedad constituyen un problema de salud a gran escala. 


\section{ISSN: 2600-5859}

Entre estas complicaciones se encuentran aquellas que aparecen en los pies, conocidas como úlceras diabéticas o síndrome del pie diabético, que conducen a una alta incidencia de amputaciones, además de los efectos secundarios negativos, como sufrimiento y costos para el paciente, su familia y la sociedad. (Marcos, 2015). La diabetes un importante problema sanitario con la presentación de complicaciones (Smith., 2016) como ceguera, alteraciones renales, neuropatía y pie diabético entre las principales (Elena, 2017)

La combinación de neuropatía, angiopatía, junto con el alto riesgo de infección y las presiones intrínseca y extrínseca debidas a las malformaciones óseas en los pies, constituyen los desencadenantes finales, del problema que en este momento nos preocupa, el pie diabético. (ALAD, 2015)

El abordaje de un problema de salud tan importante, frecuente y de no fácil solución como es el pie diabético, necesita de la atención médica a través de un equipo interdisciplinario, ya que en general son varios los profesionales sanitarios implicados en el diagnóstico y tratamiento de este síndrome, cirujanos, endocrinólogos, infectólogos, ortopedas, fisiatras, podólogos y enfermeros; del entendimiento y colaboración de todos ellos debe surgir la mejor manera de encauzarlo correctamente y con un mismo enfoque médico. (Jose, 2017)

En sus pilares de tratamiento existen numerosos aspectos, tales como son, la dieta, el tratamiento médico, el ejercicio físico y la prevención de las complicaciones agudas, crónicas y el tratamiento de las secuelas funcionales. (Lawrence M. Ternier, 2014)

La educación diabetológica y la prevención es imprescindible y fundamental, por tanto no debe ser solamente una parte importante del tratamiento, sino que, en muchos aspectos constituye el propio tratamiento o al menos la base sobre la que este debe asentarse; (Marcos, 2015) el segundo aspecto lo constituye el tratamiento médico propiamente dicho y en tercer lugar la rehabilitación integral, jugando un papel en la prevención de complicaciones y en el tratamiento de la secuelas. (Marion, 2016). La mayoría de los pacientes es de cuarenta años de edad y obesos (Mery, 2015). El síndrome de pie diabético es definido por la OMS como la ulceración, infección y/o gangrena del pie, asociados a neuropatía diabética y diferentes grados de enfermedad arterial periférica y es el resultado de la interacción compleja de diferentes factores (Orrego, 2017). Es la consecuencia de una descompensación sostenida de los valores de glicemia, que van a 
desencadenar alteraciones vasculares de la micro y macro circulación, alteraciones neuropáticas y propensión especial al padecimiento de infecciones. (Sabiston, 2016).

El problema de pie diabético como complicación de la diabetes mellitus, es uno de los más dramáticos, se ha calculado que uno de cada cinco diabéticos presentará un cuadro de pie diabético en el transcurso de su vida, una gran proporción de los pacientes que lo desarrolla están en riesgo 15 veces mayor de sufrir una complicación; por lo que 20\% requerirán amputación de la extremidad y el 30\% experimentarán una segunda amputación ipsilateral o contralateral dentro de los 12 meses posteriores y el $50 \%$ en menos de 5 años, es más frecuente después de los 40 años y se incrementan con la edad. (Sabiston, 2016) La gangrena es 71 veces más frecuente en diabéticos, que en no diabéticos. Los tiempos requeridos de hospitalización por lo general son mayores en promedio que por otras complicaciones. De todas las amputaciones $70-80 \%$ son precedidas por úlceras crónicas. (Smith., 2016)

El control de los factores de riesgo del pie diabético desempeña un papel fundamental en la reducción de la incidencia de esta afección. En el análisis de los factores de riesgo que predisponen al pie diabético hemos encontrado en estudios epidemiológicos como factores fundamentales: pacientes con diabetes mellitus con tiempo de evolución de la enfermedad superior a 10 años, de edad del paciente, especialmente en los individuos mayores de 50 años, (Stein, 2016) antecedentes de ulcera o amputación, presencia de neuropatía, artropatía o vasculopatia, presencia de otras complicaciones diabéticas bajo el nivel socio económico del paciente y aislamiento social, deficiencias en la dieta, deficiente educación en el cuidado de los pies y otros factores de riesgo asociados a la enfermedad vascular (ALAD, 2015).

Las úlceras de los pies y los problemas articulares son causas importantes de patología en la DM. La principal causa predisponente es la polineuropatía diabética; la desnervación sensitiva dificulta la percepción de los traumatismos por causas tan comunes como los zapatos mal ajustados o las piedrecillas. Las alteraciones de la sensibilidad propioceptiva conducen a un modo anormal de soportar el peso y a veces al desarrollo de articulaciones de Charcot (Orrego, 2017).

El riesgo de infección por hongos y bacterias aumenta debido a la disminución de la inmunidad celular causada por la hiperglucemia y los déficits circulatorios originados por la hiperglucemia crónica. (Orrego, 2017). El proceso inicial puede ser una infección micótica que lleva a lesiones interdigitales, grietas, fisuras y ulceraciones exudativas que facilitan una invasión 


\section{ISSN: 2600-5859}

bacteriana secundaria. Muchas veces los pacientes con úlceras de pie infectadas no sienten dolor a causa de la neuropatía y no tienen síntomas sistémicos hasta una fase posterior con una evolución desatendida. Las úlceras profundas, y en especial las úlceras asociadas con algún grado detectable de celulitis, exigen hospitalización inmediata, dado que puede aparecer toxicidad sistémica e incapacidad permanente. (Orrego, 2017)

Al igual que la arteriosclerosis, la macroangiopatía diabética tiene como característica primordial su desarrollo multifocal afectando de manera simultánea a las arterias coronarias, cerebrales, mesentéricas, renales y de las extremidades. (Lawrence M. Ternier, 2014)

La isquemia tisular condiciona un cuadro clínico en dependencia de las estructuras orgánicas cuyo aporte sanguíneo arterial resulta empobrecido; en el caso de las extremidades inferiores su expresión máxima es la gangrena, proceso consecutivo a lesiones estenooclusivas de las arterias correspondientes. (Jose, 2017)

Es frecuente la asociación de la macroangiopatía del diabético en los miembros inferiores con otras entidades como cardiopatía isquémica e insuficiencia cerebro vascular, lo que constituye un factor agravante del estado de salud de estos pacientes. (Jose, 2017)

Cuando se instauran las complicaciones crónicas en estos pacientes, estas tienen un difícil tratamiento y ocasionan graves consecuencias en la salud y calidad de vida de los pacientes y de sus familias; se estima que alrededor del $15 \%$ de todos los pacientes con diabetes mellitus desarrollará una úlcera en el pie o en la pierna durante el transcurso de su enfermedad. (Jose, 2017)

Por regla general la enfermería juega uno de los papeles primordiales en el tratamiento de las ulceraciones de los pies en el diabético, a estas las podemos enmarcar en lo que en general conocemos como heridas crónicas, es decir lesiones deprimidas de la piel con afectación variable, pudiendo llegar desde epidermis hasta plano óseo y con una característica común, y es su dificultad para cicatrizar. (Jose, 2017)

Las úlceras diabéticas constituyen un gran reto para enfermería, por el tiempo que demorar en su tratamiento y la atención mantenida, así como la gran cantidad de recursos materiales que deben emplearse en su control y curación. A su vez constituye un gran problema social, porque suponen una gran carga emocional y económica para quien las padecen, para sus familias y porque no para el sistema de salud. (Jose, 2017) 
La clasificación de las heridas crónicas ha sido siempre una necesidad de los profesionales implicados en su cuidado, pero la heterogeneidad característica de las heridas crónicas es un inconveniente a la hora de desarrollar un sistema de clasificación universal (Marcos, 2015).

Existen múltiples sistemas de clasificación de heridas crónicas. Algunos fueron diseñados para el estadiaje específico de úlceras por presión (Stein, 2016). Otros fueron diseñados específicamente para la evaluación de la efectividad de determinadas terapias (Jose, 2017). En el otro extremo existen sistemas diseñados con el fin de poder clasificar de forma universal lesiones de diferente etiología. Es el caso del sistema RYB (Moritz, 2014), que ha disfrutado de considerable difusión sobre todo entre los profesionales enfermeros (Orrego, 2017). Algunos de estos sistemas han sido utilizados frecuentemente en el estadiaje de lesiones de pie diabético. En este trabajo de investigación se señalan exclusivamente los sistemas desarrollados específicamente para su uso en lesiones de pie diabético.

\section{Metodología}

Se realizó un estudio descriptivo, retrospectivo y observacional. Se registró datos clínicos de pacientes con diabetes mellitus (DM), como el tiempo de evolución de la patología, tipo de diabetes mellitus, evolución de pie diabético y su clasificación según la escala de Wagner y Texas para lo cual se realizó examen físico minucioso en cada paciente, edad, y sexo durante el período 2018 a 2019. Se incluyó pacientes mayores de 12 años, pacientes con diagnóstico de pie diabético con o sin proceso infeccioso asociado, del Servicio de Emergencia de Cirugía General del Hospital General del Guasmo Sur de la Ciudad de Guayaquil. Los criterios de exclusión fueron pacientes referidos de otra casa de salud y con tratamiento instituido. La investigación realizada cumplió con los principios éticos de la Declaración de Helsinki.

Para la clasificación del pie diabético, luego de la examinación física minuciosa se empleó a manera de comparación la escala de Wagner, la cual clasifica al pie diabético de acuerdo a la profundidad de la úlcera, grado de infección y el grado de necrosis, así como la escala de Texas, misma que clasifica al pie diabético de acuerdo a la profundidad de la úlcera y se clasifica en estudios según la infección e isquemia.

Los datos encontrados fueron recolectados de forma física mediante la aplicación de las dos escalas mencionadas previamente, se tabuló todos los datos en hojas electrónicas de Excel, se 
ISSN: 2600-5859

procesó la información para obtención de porcentajes de variables cualitativa y cuantitativa, se empleó el índice de correlación de Pearson para cada clasificación.

\section{Resultados}

Se estudió 70 pacientes en total. Se encontró que el $60 \%$ de pacientes estudiados correspondió al género masculino y el $40 \%$ al género femenino. (Tabla 1)

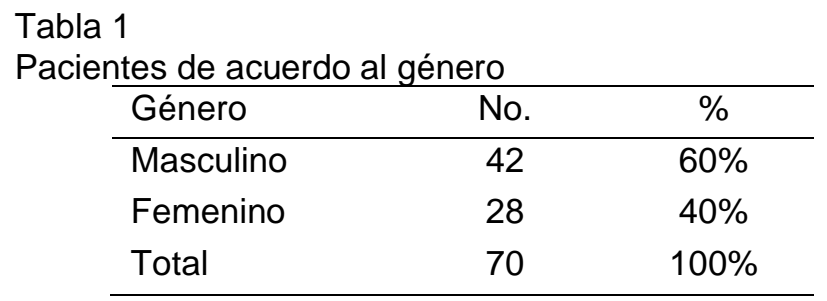

Fuente: Elaboración propia

Se clasificó a los pacientes con pie diabético de acuerdo al grupo etario, encontrándose en orden de frecuencia: $36 \%$ pacientes con edades comprendidas entre 51 a 60 años, 23\% para pacientes con edades entre 41 a 50 años, edades entre 61 a 70 años con el 23\%, $10 \%$ para pacientes entre 31 a 40 años y $3 \%$ para dos grupos etarios, entre 12 y 30 años y mayores de 70 años. (Tabla 2)

Tabla 2

Grupos etarios de pacientes con pie diabético.

\begin{tabular}{ccc}
\hline Rango de edades & No. & $\%$ \\
\hline $12-30$ años & 3 & $4 \%$ \\
$31-40$ años & 7 & $10 \%$ \\
$41-50$ años & 16 & $23 \%$ \\
$51-60$ años & 25 & $36 \%$ \\
$61-70$ años & 16 & $23 \%$ \\
$>$ de 70 años & 3 & $4 \%$ \\
Total & 70 & $100 \%$ \\
\hline
\end{tabular}

Fuente: Elaboración propia

De acuerdo al tipo de diabetes mellitus, el $14 \%$ de pacientes fue tipo 1, y el $40 \%$ fue tipo 2 , de acuerdo al género y tipo de diabetes, 2 pacientes masculinos y 8 femeninos para el tipo 1; 40 pacientes masculinos (M) y 28 femeninos (F) para el tipo de diabetes mellitus 2. (Tabla 3) 
ISSN: 2600-5859

Tabla 3

Tipo de diabetes mellitus de acuerdo al género

\begin{tabular}{ccccc}
\hline Tipo de diabetes mellitus & No. & $\%$ & M & F \\
\hline Tipo I & 10 & 14 & 2 & 8 \\
Tipo II & 60 & 86 & 40 & 20 \\
Total & 70 & $100 \%$ & 42 & 28 \\
\hline
\end{tabular}

Fuente: Elaboración propia

De acuerdo al tiempo de evolución de las lesiones del pie diabético desde el inicio de la enfermedad, se encontró, en orden descendente de frecuencia: evolución de 16 a 20 años con el $36 \%$, seguido de 11 a 15 años de evolución con el 34\%, evolución mayor de 20 años con el 16\%, de 5 a 10 años de evolución con el 11\% y evolución menor de 5 años con el 3\%. (Tabla 4).

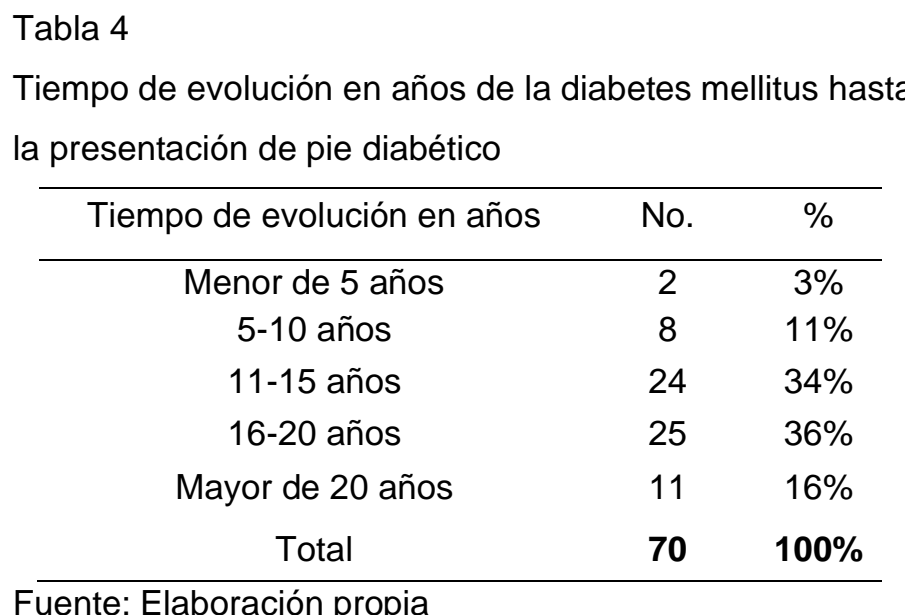

A la examinación física minuciosa se encontró diversas características clínicas, según la escala de Texas, de acuerdo a los grados predominó el 3 con el 46\% de pacientes, luego el grado 2 con el $40 \%$ y finalmente el grado 1 con 14\%, de los cuales el grado 3 presentó mayor porcentaje (72\%) para amputación, para cicatrización el grado 2 con el 43\%, para no cicatrización con el 40\% en el grado 1 y mayor prevalencia de mortalidad en el grado 3 con el 3\%. (Tabla 5) 
ISSN: 2600-5859

Tabla 5

Grado de pie diabético según la escala de Universidad de Texas y su evolución

\begin{tabular}{|c|c|c|c|c|c|c|c|c|c|c|c|}
\hline \multirow[t]{2}{*}{$\begin{array}{l}\text { ETAPA Y } \\
\text { GRADOS }\end{array}$} & \multirow[b]{2}{*}{$\begin{array}{l}\text { No. De } \\
\text { pacientes }\end{array}$} & \multirow[b]{2}{*}{$\begin{array}{l}\% \text { por } \\
\text { etapas }\end{array}$} & \multicolumn{2}{|c|}{ Amputación } & \multicolumn{2}{|c|}{ Cicatrizaron } & \multicolumn{2}{|c|}{$\begin{array}{l}\text { No } \\
\text { cicatrizaron }\end{array}$} & \multicolumn{2}{|c|}{ Fallecieron } & \multirow{2}{*}{\begin{tabular}{|c|} 
TOTAL \\
$\%$
\end{tabular}} \\
\hline & & & No. ptes & $\%$ & No. ptes & $\%$ & No. ptes & $\%$ & No ptes & $\%$ & \\
\hline GRADO 1 & 10 & $14 \%$ & 3 & $30 \%$ & 3 & $30 \%$ & 4 & $40 \%$ & . & - & $100 \%$ \\
\hline ETAPA A & 1 & & - & - & 1 & $100 \%$ & - & - & . & - & $100 \%$ \\
\hline ETAPA B & 0 & & - & - & 0 & - & - & - & . & - & - \\
\hline ETAPAC & 5 & & 1 & $20 \%$ & 1 & $20 \% \%$ & 3 & $60 \%$ & . & - & $100 \%$ \\
\hline ETAPA D & 4 & & 2 & $50 \%$ & 1 & $25 \%$ & 1 & $25 \%$ & 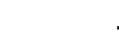 & - & $100 \%$ \\
\hline GRADO 2 & 28 & $40 \%$ & 13 & $46 \%$ & 12 & $43 \%$ & 3 & $11 \%$ & . & - & $100 \%$ \\
\hline ETAPA A & 3 & & - & - & 3 & $100 \%$ & - & - & 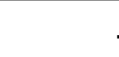 & - & $100 \%$ \\
\hline ETAPA B & 3 & & - & - & 3 & $100 \%$ & - & - & . & - & $100 \%$ \\
\hline ETAPA C & 7 & & 1 & $14 \%$ & 5 & $72 \%$ & 1 & $14 \%$ & . & - & $100 \%$ \\
\hline ETAPA D & 15 & & 12 & $80 \%$ & 1 & $7 \%$ & 2 & $13 \%$ & . & - & $100 \%$ \\
\hline GRADO 3 & 32 & $46 \%$ & 23 & $72 \%$ & 7 & $22 \%$ & 1 & $3 \%$ & & $3 \%$ & $100 \%$ \\
\hline ETAPA A & 1 & & - & - & 1 & $100 \%$ & - & - & . & - & $100 \%$ \\
\hline ETAPA B & 4 & & 1 & $25 \%$ & 3 & $75 \%$ & - & - & 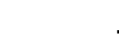 & - & $100 \%$ \\
\hline ETAPAC & 8 & & 4 & $50 \%$ & 3 & $37 \%$ & 1 & $13 \%$ & . & - & $100 \%$ \\
\hline ETAPA D & 19 & & 18 & $95 \%$ & 0 & - & - & - & & $5 \%$ & $100 \%$ \\
\hline TOTAL & 70 & $100 \%$ & 39 & $56 \%$ & 22 & $31 \%$ & 8 & $12 \%$ & 1 & $1 \%$ & $100 \%$ \\
\hline
\end{tabular}

Fuente: Elaboración propia

Según la escala de Wagner, se encontró con mayor prevalencia el grado 5 con el $66 \%$ para amputaciones, el grado 1 para cicatrización con el 100\%, el grado 3 y grado 5 para no cicatrización con el 17\% respectivamente, y el grado 5 con el 17\% para mortalidad. (Tabla 6) 
ISSN: 2600-5859

Tabla 6

Grado de pie diabético según la escala de Wagner y su evolución

\begin{tabular}{|c|c|c|c|c|c|c|c|c|c|c|c|}
\hline \multirow[t]{3}{*}{$\begin{array}{l}\text { GRADO DE PIE } \\
\text { DIABE ICO }\end{array}$} & \multirow{3}{*}{ No. ptes } & \multirow{3}{*}{\begin{tabular}{|c|}
$\%$ \\
ptes
\end{tabular}} & \multicolumn{2}{|c|}{ AMPUT ACIONES } & \multicolumn{2}{|c|}{ CICATRIZARON } & \multicolumn{2}{|c|}{$\begin{array}{l}\text { NO } \\
\text { CICATRIZARON }\end{array}$} & \multicolumn{2}{|c|}{ FALLECIERON } & \multirow{3}{*}{$\begin{array}{c}\text { TOTAL } \\
\%\end{array}$} \\
\hline & & & \multirow{2}{*}{ No. } & \multirow[t]{2}{*}{$\%$} & \multirow[t]{2}{*}{ №. } & \multirow[t]{2}{*}{$\%$} & \multirow[t]{2}{*}{ No. } & \multirow[t]{2}{*}{$\%$} & \multirow[t]{2}{*}{ No. } & \multirow[t]{2}{*}{$\%$} & \\
\hline & & & & & & & & & & & \\
\hline GRADO 1 & 4 & $6 \%$ & - & - & 4 & $100 \%$ & - & - & - & - & $100 \%$ \\
\hline GRADO 2 & 9 & $13 \%$ & 1 & $11 \%$ & 7 & $78 \%$ & 1 & $\#$ & - & - & $100 \%$ \\
\hline GRADO 3 & 30 & $43 \%$ & 19 & $63 \%$ & 6 & $20 \%$ & 5 & \# & - & - & $100 \%$ \\
\hline GRADO 4 & 21 & $30 \%$ & 15 & $71 \%$ & 5 & $24 \%$ & 1 & $5 \%$ & - & - & $100 \%$ \\
\hline GRADO 5 & 6 & $8 \%$ & 4 & $66 \%$ & - & - & 1 & $\#$ & 1 & $17 \%$ & $100 \%$ \\
\hline TOTAL & 70 & $100 \%$ & 39 & $56 \%$ & 22 & $31 \%$ & 8 & \#\# & 1 & $1 \%$ & $100 \%$ \\
\hline
\end{tabular}

Fuente: Elaboración propia

\section{Conclusiones}

Los tres componentes patológicos, neuropatía, enfermedad vascular periférica y deformidades óseas, sobre los que gira la etiopatogenia de las lesiones que se desencadenan en el pie del diabético pueden traer consecuencias graves a estos pacientes. Una exploración detallada puede dar claves para diagnosticar la enfermedad o para confirmar el avanzado estado de esta. El control de la diabetes y el tratamiento adecuado de las úlceras, infecciones, deformidades músculo esqueléticas, proporcionará al paciente una mejor calidad de vida.

El papel del profesional de la salud es saber abordar este grave problema de salud; la educación sanitaria diabetológica, las recomendaciones de prevención, la identificación de riesgos, el establecimiento de un plan de cuidados individualizado y el curetaje local de las úlceras constituyen los pilares sobre los que se asienta este rol.

A pesar de la efectividad de diferentes medios y agentes terapéuticos es necesario seguir investigando y haciendo las pertinentes evaluaciones clínicas de las nuevas terapias, para conseguir cada vez productos más eficaces que aceleren los procesos de cicatrización en las úlceras del pie diabético. El reto para el equipo interdisciplinario es enfrentarse a estas lesiones con la suficiente preparación $\mathrm{v}$ y conocimientos profesionales, y usar los productos a su alcance 
ISSN: 2600-5859

de la manera más adecuada, para dar los mejores cuidados posibles, que restauren la salud del diabético y restablezcan en el menor tiempo posible la integridad de la piel, evitando así el mayor número posible de amputaciones.

\section{Referencias bibliográficas}

ALAD. (2015). Consejo Sobre Prevención, Control, Tratamiento De La Diabetes Mellitus No Insulinodependiente. México: ALAD.

Elena, A. C. (2017). Diagnóstico y Tratamiento. Madrid: Elsevier.

González de la Torre, H. P. (2016). Estratificación de riesgo en pie diabético. Gerokomos, 21(4), $172-182$

INEC. (2013). Información histórica, nacimientos y defunciones. Guayaquil: Instituto Nacional de estadísticas y censos

INEC. (2014). Principales causas de mortalidad. Guayaquil: Instituto Nacional de estadísticas y censos.

Jacobsen, I. H. Evidence. Based insulin treatment in type 1 Diabetes Mellitus. Diabetes Res Clin Pact, 86(1), 1-10.

Jara, A. Endocrinología. España: Panamericana S.A.

Jarrín, J. Y. Pie Diabético; Tratamiento Local Del Pie Diabético. Quito.

Jiménez, A. I. Identificación del riesgo de pie diabético y factores asociados. Enfermería Clínica, 15(5), 283-286.

Jiménez, F. T. La diabetes mellitus en la práctica clínica. Madrid: Panamericana.

José, M. M. (2017). Pie Diabético. Buenos Aires: Alfa Omega.

Khuwaja, A. R. Macrovascular complications and either associated factors amnog persons with type 2 diabetes in Karachi, Pakistan a multicenter study. J Pak Med Assoc, 54(2), 60-66.

Kumar, S. A. The prevalence of foot ulceration and its correlates in type 2 diabetic patients: A population-based study. Diabetes Med, 11(5), 480-484.

Lawrence M. Ternier, J. S. (2014). Diagnóstico Clínico Y Tratamiento. Madrid: Manual Moderno.

MSP. Anuario de vigilancia epidemiológica de enfermedades crónicas 1994-2012. Cuenca: Ministerio de Salud Pública. 
ISSN: 2600-5859

Marcos, L. G. (2015). Tratado de Medicina Interna. Guadalajara: Elsevier.

Marion, M. G. (2016). Medicina Interna. Cuba: La Habana.

Mery, C. Z. (2015). Diagnóstico y Tratamiento de Diabetes Mellitus Tipo II. Barcelona: Abmco.

Moritz, G. F. (2014). Endocrinología, enfoque desde la fisiopatología a la terapéutica. Berlín.: Data Becker.

Nathan, D. B. Management of hyperglycemia in type 2 diabetes: A consensus algorithm for the initiation and adjustment of therapy: a consensus statement from the American Diabetes Association and the European Association for the Study of Diabetes. Diabetes Care, 29(8), 1963-1972.

Núñez, B. Infecciones en el pie diabético. En Núñez-Freile, Pie diabético, manual de tratamiento clínico quirúrgico (1ºd., págs. 101-127). Quito, Pichincha, Ecuador: Elsevier.

OMS. (2015). Diabetes, Nota descriptiva n³12. OMS: OMS.

OPS. (2014). La diabetes, un problema prioritario de salud pública en el Ecuador y la región de las Américas. Colombia: Organización Panamericana de la salud.

Orrego, A. (2017). Endocrinología. Fundamentos de Medicina. Canarias: Adeka.

Sabiston, C. (2016). Tratado De Patología Quirúrgica, Bases Biológicas De La Práctica Quirúrgica. Washington DC.: Moderna.

Smith., C. W. (2016). Tratado De Medicina Interna. Nevada: Elsevier.

Stein, J. (2016). Medicina Interna. Washington DC: Manual Moderno.

Vaquero, C. (2015). Pie diabético. Valladolid.

Valk, G. D. Educación de los pacientes para la prevención de la ulceración del pie diabético. Biblioteca Cochrane Plus.

Stockl, K. V. Costs of lower-extremity ulcers among patients with diabetes. Diabetes Care, 27(9), 2129-2134

Skamagas, M. B. Update on Diabetes Mellitus: prevention, treatment, and association with oral diseases. Oral Dis, 14(2), 105-114.

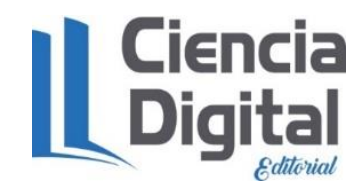


ISSN: 2600-5859

\section{PARA CITAR EL ARTÍCULO INDEXADO}

Cervantes Moyano, K. P., Delgado Rivera, A. E., Reyes Murillo, K. K., \& Veloz Estrada, L. S. (2020). Estratificación del pie diabético en el Hospital General Guasmo Sur período 2018 - 2019. ConcienciaDigital, 3(1.2), 20-32. https://doi.org/10.33262/concienciadigital.v3i1.2.1167

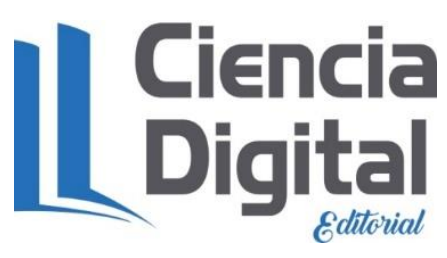

El artículo que se publica es de exclusiva responsabilidad de los autores y no necesariamente reflejan el pensamiento de la Revista Conciencia Digital.

El artículo queda en propiedad de la revista y, por tanto, su publicación parcial y/o total en otro medio tiene que ser autorizado por el director de la Revista Conciencia Digital.
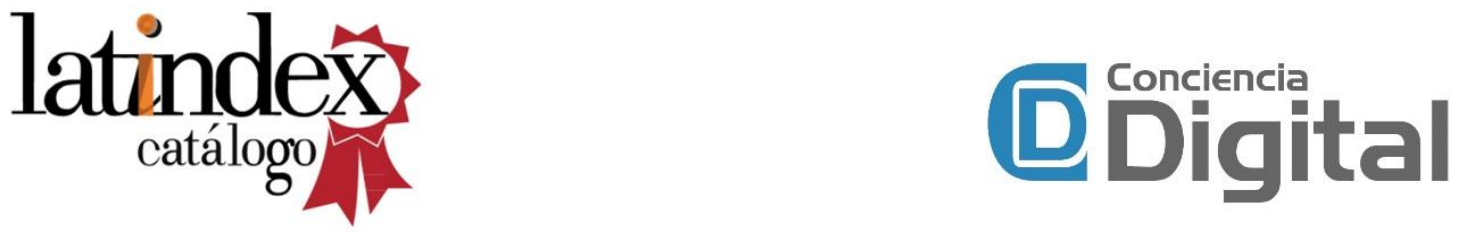